\title{
Influence of spatial inhomogeneity of environment temperature on temperature oscillations and thermocyclic stresses in elastic half-space
}

\author{
Maxim Supel'nyak* \\ Bauman Moscow State Technical University (Kaluga Branch), Kaluga, Russia
}

\begin{abstract}
Elastic half-space on which surface the heat transfer follows the Newton-Richmann law where enviroment temperature is a periodic function of time and spatial variable is investigated. A temperature field and a stressed deformed state of half-space is found after the solution of a quasistatic problem of thermoelasticity. The solution is received in the form of double Fourier series.
\end{abstract}

\section{Problem definition}

An important place in thermal conductivity and thermoelasticity researches is occupied by problems concerning temperature oscillations of a solid and thermocyclic stresses caused by them. The relevance of this type processes studying is connected with the fact that they proceed in the Earth crust [1], external walls of buildings [2, 3], blades of gas turbines [4], elements of internal combustion engines [5], regenerative heat exchangers [6], and find an application when determining coefficients of thermal conductivity and thermal diffusivity [1] and some other material properties [7, 8].

Initial studies of thermal conductivity and thermoelasticity periodic processes were conducted for an environment model based on Fourier's and Hooke's phenomenological laws under the simplest types of boundary conditions. Periodic solutions of classical problems of thermal conductivity for solids of canonical form are provided in [1], and the relevant solutions of classical problems of thermoelasticity can be found in $[9,10]$. Follow up study of thermal conductivity and thermoelasticity cyclic processes was conducted in two directions. Researches in which the classical models of environments were used and which are based on Fourier's and Hooke's phenomenological laws, but the problem of thermal conductivity was solved with boundary conditions of more difficult type, which considered real features of the process, belong to the first direction. In research works [11, $12,13]$ and some other ones heat transfer coefficient was accepted as non-stationary, and in [11] the solution of heat conductivity problem is received at an arbitrary periodic dependence of heat transfer coefficient on time. In some researches the dependence of thermophysical properties of environment on temperature was considered. In [14] the

*Corresponding author: max2901@ mail.ru 
nonlinear equation of thermal conductivity with nonlinear boundary conditions was solved. The second direction of research was developing in parallel with development of some new thermomechanical environment models eliminating paradoxes of classical thermoelastic model. Early researches in this direction are connected with use of thermoviscoelastic environment model [9]. Further also other models were developed by which using thermal waves and thermocyclic stresses were investigated. For example, in [15, 16] thermoelasticity without energy dissipation theory which is offered in [17] was used. Various thermomechanical environments are considered in fundamental work [18]. In [19] thermal waves in environments with dual-phase-lagging are studied. In the last decades in connection with progress in development of computer facilities there was an opportunity to solve numerically various boundary problems in areas of irregular shape and with variable properties of the environment. It is possible to give as works examples [20, 21] where thermal conductivity and thermomechanical cyclic processes were numerically investigated.

In the majority of theoretical research works of temperature oscillations and thermocyclic stresses the thermal conductivity and thermoelasticity problems were solved in one-dimensional statement. Such approach is usually fair when temperature oscillations in a solid don't extend to all depth, and decay in a thin surface layer called thermal one [22]. At the same time in actual practice a spatial nonuniformity of heat transfer conditions takes place because of which one-dimensional solutions can become doubtful. In such situation it is necessary to use two-dimensional or three-dimensional solution of the thermoelasticity problem. Particular two-dimensional cyclic problems of heat conductivity for a plate have been considered in works $[12,13]$.

The purpose of the undertaken study was the establishment of influence of spatial inhomogeneity of heat transfer conditions on temperature oscillations and thermocyclic stresses in a thermal layer. For this purpose an elastic half-space on which surface heat transfer follows the Newton-Richmann law where environment temperature is a periodic function of time and spatial variable was investigated.

\subsection{Mathematical model}

For convenience we will proceed to dimensionless variables

$$
\begin{gathered}
\hat{x}=\frac{x}{\delta}, \hat{y}=\frac{2 \pi y}{l}, \hat{l}=\frac{l}{2 \pi \delta}, \hat{t}=\omega t, \mathrm{Bi}=\frac{\alpha \delta}{\lambda}, \hat{\vartheta}=\frac{T-\left\langle T_{f}^{*}\right\rangle}{\Delta T_{f}^{*}}, \hat{\vartheta}_{f}=\frac{T_{f}-\left\langle T_{f}^{*}\right\rangle}{\Delta T_{f}^{*}}, \\
\hat{u}_{x}=\frac{u_{x}}{\delta}, \hat{u}_{y}=\frac{u_{y}}{\delta}, \hat{\sigma}_{x x}=\frac{\sigma_{x x}}{E}, \hat{\sigma}_{y y}=\frac{\sigma_{y y}}{E}, \hat{\sigma}_{z z}=\frac{\sigma_{z z}}{E}, \hat{\sigma}_{x y}=\frac{\sigma_{x y}}{E}, \\
\varepsilon_{\theta}=\Delta \varepsilon_{T_{f}^{*}} \hat{\theta}, \hat{\theta}=\hat{\vartheta}-\langle\hat{\vartheta}\rangle_{\hat{y}, \hat{t}}, \Delta \varepsilon_{T_{f}^{*}}=\beta \Delta T_{f}^{*},
\end{gathered}
$$

where $x, y$ - spatial coordinates, $\mathrm{m} ; \delta=\sqrt{a / \omega}$ - representative depth of temperature waves penetration, $\mathrm{m} ; l$ - spatial period, $\mathrm{m} ; t$ - time, $\mathrm{s} ; \omega=2 \pi / \mathcal{T}$ - oscillation angular frequency, $\mathrm{s}^{-1} ; \mathcal{T}$ - oscillation period, $\mathrm{s} ; \alpha$ - heat transfer coefficient, $\mathrm{W} /\left(\mathrm{m}^{2} \cdot \mathrm{K}\right) ; \lambda-$ heat conduction coefficient, $\mathrm{W} /(\mathrm{m} \cdot \mathrm{K}) ; T=T(\hat{x}, \hat{y}, \hat{t})$ - temperature of half-space, $\mathrm{K}$; $T_{f}=T_{f}(\hat{y}, \hat{t})$ - temperature of fluid, $\mathrm{K} ;\left\langle T_{f}^{*}\right\rangle$ - representative oscillation period averaged temperature of fluid, $\mathrm{K} ; \Delta T_{f}^{*}$ - representative temperature of fluid range, $\mathrm{K} ; u_{x}, u_{y}$ components of displacement vector $\mathbf{u}=\mathbf{u}(\hat{x}, \hat{y}, \hat{t}), \mathrm{m} ; \mathbf{u}=u_{x} \mathbf{i}+u_{y} \mathbf{j}-$ displacement vector, $\mathrm{m} ; \sigma_{x x}, \sigma_{y y}, \sigma_{z z}, \sigma_{x y}-$ components of stress tensor $\mathrm{T}_{\sigma}=\mathrm{T}_{\sigma}(\hat{x}, \hat{y}, \hat{t}), \mathrm{Pa}$;

$$
\mathrm{T}_{\sigma}=\left(\begin{array}{ccc}
\sigma_{x x} & \sigma_{x y} & 0 \\
\sigma_{x y} & \sigma_{y y} & 0 \\
0 & 0 & \sigma_{z z}
\end{array}\right)
$$


- stress tensor, Pa; $E$ - Young modulus, $\mathrm{Pa} ;\langle\hat{\vartheta}\rangle_{\hat{y}, \hat{t}}$ - dimensionless excess temperature of half-space averaged over oscillation period and spatial period; $\beta$-coefficient of linear thermal expansion, $\mathrm{K}^{-1}$.

We will consider the $T_{f}(\hat{y}, \hat{t})$ functions, periodic in $\hat{y}$ and $\hat{t}$ with $2 \pi$ periods for which $T_{f}(\hat{y}+2 \pi, \hat{t})=T_{f}(\hat{y}, \hat{t}+2 \pi)=T_{f}(\hat{y}, \hat{t}),-\infty<\hat{y}<+\infty, \hat{t}>-\infty$.

Under such condition all the required dependences, after the process changes to the steadystate oscillation regime, will also be periodic in $\hat{y}$ and $\hat{t}$ functions:

$$
\begin{aligned}
T(\hat{x}, \hat{y}+2 \pi, \hat{t}) & =T(\hat{x}, \hat{y}, \hat{t}+2 \pi)=T(\hat{x}, \hat{y}, \hat{t}), \hat{x} \geq 0,-\infty<\hat{y}<+\infty, \hat{t}>-\infty \\
\mathbf{u}(\hat{x}, \hat{y}+2 \pi, \hat{t}) & =\mathbf{u}(\hat{x}, \hat{y}, \hat{t}+2 \pi)=\mathbf{u}(\hat{x}, \hat{y}, \hat{t}), \hat{x} \geq 0,-\infty<\hat{y}<+\infty, \hat{t}>-\infty \\
\mathrm{T}_{\sigma}(\hat{x}, \hat{y}+2 \pi, \hat{t}) & =\mathrm{T}_{\sigma}(\hat{x}, \hat{y}, \hat{t}+2 \pi)=\mathrm{T}_{\sigma}(\hat{x}, \hat{y}, \hat{t}), \hat{x} \geq 0,-\infty<\hat{y}<+\infty, \hat{t}>-\infty
\end{aligned}
$$

To evaluate them it is necessary to solve the uncoupled quasistatic problem of thermoelasticity [4].

Having transformed a boundary problem of heat conduction [1] into a dimensionless form, we will receive the system of equations

$$
\begin{gathered}
\frac{\partial \hat{\vartheta}}{\partial \hat{t}}=\frac{\partial^{2} \hat{\vartheta}}{\partial \hat{x}^{2}}+\frac{1}{\hat{l}^{2}} \frac{\partial^{2} \hat{\vartheta}}{\partial \hat{y}^{2}}, \hat{x}>0,-\infty<\hat{y}<+\infty, \hat{t}>-\infty \\
\frac{\partial \hat{\vartheta}(0, \hat{y}, \hat{t})}{\partial \hat{x}}=\operatorname{Bi}\left[\hat{\vartheta}(0, \hat{y}, \hat{t})-\hat{\vartheta}_{f}(\hat{y}, \hat{t})\right],-\infty<\hat{y}<+\infty, \hat{t}>-\infty
\end{gathered}
$$

$\hat{\vartheta}(\hat{x}, \hat{y}+2 \pi, \hat{t})=\hat{\vartheta}(\hat{x}, \hat{y}, \hat{t}+2 \pi)=\hat{\vartheta}(\hat{x}, \hat{y}, \hat{t}), \hat{x} \geq 0,-\infty<\hat{y}<+\infty, \hat{t}>-\infty$. (3) Having transformed a boundary problem of elasticity theory [4] into a dimensionless form, we will receive the system of equations

$$
\begin{aligned}
& \begin{array}{c}
\frac{2(1-v)}{1-2 v} \frac{\partial^{2} \hat{u}_{x}}{\partial \hat{x}^{2}}+\frac{1}{\hat{l}^{2}} \frac{\partial^{2} \hat{u}_{x}}{\partial \hat{y}^{2}}+\frac{1}{1-2 v} \frac{1}{\hat{l}} \frac{\partial^{2} \hat{u}_{y}}{\partial \hat{x} \partial \hat{y}}=\frac{2(1+v)}{1-2 v} \frac{\partial \varepsilon_{\theta}}{\partial \hat{x}}, \\
\hat{x}>0,-\infty<\hat{y}<+\infty, \hat{t}>-\infty ; \\
\begin{array}{c}
\frac{\partial^{2} \hat{u}_{y}}{\partial \hat{x}^{2}}+\frac{2(1-v)}{1-2 v} \frac{1}{\hat{l}^{2}} \frac{\partial^{2} \hat{u}_{y}}{\partial \hat{y}^{2}}+\frac{1}{1-2 v} \frac{1}{\hat{l}} \frac{\partial^{2} \hat{u}_{x}}{\partial \hat{x} \partial \hat{y}}=\frac{2(1+v)}{1-2 v} \frac{1}{\hat{l}} \frac{\partial \varepsilon_{\theta}}{\partial \hat{y}}, \\
\hat{x}>0,-\infty<\hat{y}<+\infty, \hat{t}>-\infty ; \\
\begin{array}{r}
(1-v) \frac{\partial \hat{u}_{x}(0, \hat{y}, \hat{t})}{\partial \hat{x}}+v \frac{1}{\hat{l}} \frac{\partial \hat{u}_{y}(0, \hat{y}, \hat{t})}{\partial \hat{y}}=(1+v) \varepsilon_{\theta}(0, \hat{y}, \hat{t}), \\
\frac{1 \partial \hat{u}_{x}(0, \hat{y}, \hat{t})}{\partial \hat{y}}+\frac{\partial \hat{u}_{y}(0, \hat{y}, \hat{t})}{\partial \hat{x}}=0,-\infty<\hat{y}<+\infty, \hat{t}>-\infty ; \\
\lim _{\hat{x} \rightarrow+\infty} \hat{u}_{x}=0,-\infty<\hat{y}<+\infty, \hat{t}>-\infty ; \\
\lim _{\hat{x} \rightarrow+\infty} \hat{u}_{y}=0,-\infty<\hat{y}<+\infty, \hat{t}>-\infty ;
\end{array} \\
\hat{u}_{x}(\hat{x}, \hat{y}+2 \pi, \hat{t})=\hat{u}_{x}(\hat{x}, \hat{y}, \hat{t}+2 \pi)=\hat{u}_{x}(\hat{x}, \hat{y}, \hat{t}), \\
\hat{x} \geq 0,-\infty<\hat{y}<+\infty, \hat{t}>-\infty ;
\end{array} \\
\hat{u}_{y}(\hat{x}, \hat{y}+2 \pi, \hat{t})=\hat{u}_{y}(\hat{x}, \hat{y}, \hat{t}+2 \pi)=\hat{u}_{y}(\hat{x}, \hat{y}, \hat{t}), \\
\hat{x} \geq 0,-\infty<\hat{y}<+\infty, \hat{t}>-\infty .
\end{array}
\end{aligned}
$$

Here $v$ is Poisson ratio. Dimensionless components of stress tensor are determined after solving for the field of dimensionless displacement by formulas

$$
\begin{gathered}
\hat{\sigma}_{x x}=\frac{1}{(1+v)(1-2 v)}\left[(1-v) \frac{\partial \hat{u}_{x}}{\partial \hat{x}}+v \frac{1}{\hat{l}} \frac{\partial \hat{u}_{y}}{\partial \hat{y}}-(1+v) \varepsilon_{\theta}\right] \\
\hat{\sigma}_{y y}=\frac{1}{(1+v)(1-2 v)}\left[v \frac{\partial \hat{u}_{x}}{\partial \hat{x}}+(1-v) \frac{1}{\hat{l}} \frac{\partial \hat{u}_{y}}{\partial \hat{y}}-(1+v) \varepsilon_{\theta}\right] ; \\
\hat{\sigma}_{z z}=\frac{1}{(1+v)(1-2 v)}\left[v \frac{\partial \hat{u}_{x}}{\partial \hat{x}}+v \frac{1}{\hat{\imath}} \frac{\partial \hat{u}_{y}}{\partial \hat{y}}-(1+v) \varepsilon_{\theta}\right] ;
\end{gathered}
$$




$$
\hat{\sigma}_{x y}=\frac{1}{2(1+v)}\left(\frac{1}{\hat{l}} \frac{\partial \hat{u}_{x}}{\partial \hat{y}}+\frac{\partial \hat{u}_{y}}{\partial \hat{x}}\right) .
$$

\section{Solution of the problem}

As all the required dependences are periodic in $\hat{y}$ and $\hat{t}$ functions, they can be presented in the form of double Fourier series [5]. Expansion of function $f=f(\hat{y}, \hat{t})$, which is periodic in $\hat{y}$ and $\hat{t}$ with $2 \pi$ periods, in Fourier series we will write down in a complex form

$$
f=\sum_{m=-\infty}^{m=+\infty} \sum_{n=-\infty}^{n=+\infty} \mathcal{C}_{m, n}^{f} e^{i(m \hat{y}+n \hat{t})},
$$

where

$$
\mathcal{C}_{m, n}^{f}=\frac{1}{4 \pi^{2}} \int_{0}^{2 \pi} \int_{0}^{2 \pi} f e^{-i(m \hat{y}+n \hat{t})} d \hat{y} d \hat{t} .
$$

Use of the Fourier series complex form facilitates considerably operations with series, and transition to the real-valued form

$$
\begin{aligned}
f=\frac{A_{0,0}^{f}}{4}+\frac{1}{2} \sum_{m=1}^{+\infty}\left[A_{m, 0}^{f} \cos (m \hat{y})+B_{m, 0}^{f} \sin (m \hat{y})\right]+ \\
+\frac{1}{2} \sum_{n=1}^{+\infty}\left[A_{0, n}^{f} \cos (n \hat{t})+C_{0, n}^{f} \sin (n \hat{t})\right]+\sum_{m=1}^{+\infty} \sum_{n=1}^{+\infty}\left[A_{m, n}^{f} \cos (m \hat{y}) \cos (n \hat{t})+\right. \\
\left.+B_{m, n}^{f} \sin (m \hat{y}) \cos (n \hat{t})+C_{m, n}^{f} \cos (m \hat{y}) \sin (n \hat{t})+D_{m, n}^{f} \sin (m \hat{y}) \sin (n \hat{t})\right]
\end{aligned}
$$

can be made without any difficulties, having taken into account dependences

$$
\begin{gathered}
A_{m, n}^{f}=\frac{1}{\pi^{2}} \int_{0}^{2 \pi} \int_{0}^{2 \pi} f \cos (m \hat{y}) \cos (n \hat{t}) d \hat{y} d \hat{t}, B_{m, n}^{f}=\frac{1}{\pi^{2}} \int_{0}^{2 \pi} \int_{0}^{2 \pi} f \sin (m \hat{y}) \cos (n \hat{t}) d \hat{y} d \hat{t}, \\
C_{m, n}^{f}=\frac{1}{\pi^{2}} \int_{0}^{2 \pi} \int_{0}^{2 \pi} f \cos (m \hat{y}) \sin (n \hat{t}) d \hat{y} d \hat{t}, D_{m, n}^{f}=\frac{1}{\pi^{2}} \int_{0}^{2 \pi} \int_{0}^{2 \pi} f \sin (m \hat{y}) \sin (n \hat{t}) d \hat{y} d \hat{t}, \\
\mathcal{C}_{m, n}^{f}=\frac{A_{m, n}^{f}-i B_{m, n}^{f}-i C_{m, n}^{f}-D_{m, n}^{f}}{4} .
\end{gathered}
$$

By means of finite complex Fourier transformation in $\hat{y}$ and $\hat{t}$ variables we will change from the system of equations (1) - (15) for required functions to the system of equations for coefficients of their Fourier series. Instead of (1) - (11) we will receive a boundary problem

$$
\begin{gathered}
\frac{d^{2} \mathcal{C}_{m, n}^{T}}{d \hat{x}^{2}}-\left(\frac{m^{2}}{\hat{l}^{2}}+i n\right) \mathcal{C}_{m, n}^{T}=0, \hat{x}>0, m \in \mathbb{Z}, n \in \mathbb{Z} ; \\
-\frac{d \mathcal{C}_{m, n}^{T}(0)}{d \hat{x}}+\mathrm{Bi}_{m, n}^{T}(0)=\mathrm{Bi}_{m, n}^{T_{f}}, m \in \mathbb{Z}, n \in \mathbb{Z} ; \\
\frac{2(1-v)}{1-2 v} \frac{d^{2} \mathcal{C}_{m, n}^{\hat{u}_{x}}}{d \hat{x}^{2}}-\frac{m^{2}}{\hat{l}^{2}} \mathcal{C}_{m, n}^{\hat{u}_{x}}+i \frac{1}{1-2 v} \frac{m}{\hat{l}} \frac{d \mathcal{C}_{m, n}^{\widehat{u}_{y}}}{d \hat{x}}=\frac{2(1+v)}{1-2 v} \frac{d \mathcal{C}_{m, n}^{\varepsilon_{\theta}}}{d \hat{x}}, \\
\frac{d^{2} \mathcal{C}_{m, n}^{\hat{u}_{y}}}{d \hat{x}^{2}}-\frac{2(1-v)}{1-2 v} \frac{m^{2}}{\hat{l}^{2}} \mathcal{C}_{m, n}^{\widehat{u}_{y}}+i \frac{1}{1-2 v} \frac{m}{\hat{l}} \frac{d \mathcal{C}_{m, n}^{\widehat{u}_{x}}}{d \hat{x}}=i \frac{2(1+v)}{1-2 v} \frac{m}{\hat{l}} \mathcal{C}_{m, n}^{\varepsilon_{\theta}}, \\
(1-v) \frac{d \mathcal{C}_{m, n}^{\hat{u}_{x}}(0)}{d \hat{x}}+i v \frac{m}{\hat{l}} \mathcal{C}_{m, n}^{\widehat{u}_{y}}(0)=(1+v) \mathcal{C}_{m, n}^{\varepsilon_{\theta}}(0), m \in \mathbb{Z}, n \in \mathbb{Z} ;
\end{gathered}
$$




$$
\begin{gathered}
i \frac{m}{\hat{l}} \mathcal{C}_{m, n}^{\widehat{u}_{x}}(0)+\frac{d \mathcal{C}_{m, n}^{\widehat{u}_{y}}(0)}{d \hat{x}}=0, m \in \mathbb{Z}, n \in \mathbb{Z} ; \\
\lim _{\hat{x} \rightarrow+\infty} \mathcal{C}_{m, n}^{\widehat{u}_{x}}=0, m \in \mathbb{Z}, n \in \mathbb{Z} ; \\
\lim _{\hat{x} \rightarrow+\infty} \mathcal{C}_{m, n}^{\widehat{u}_{y}}=0, m \in \mathbb{Z}, n \in \mathbb{Z} .
\end{gathered}
$$

Here $\mathbb{Z}$ is a set of integers. Instead of (12) - (15) we will receive dependences

$$
\begin{gathered}
\mathcal{C}_{m, n}^{\widehat{\sigma}_{x x}}=\frac{1}{(1+v)(1-2 v)}\left[(1-v) \frac{d \mathcal{C}_{m, n}^{\hat{u}_{x}}}{d \hat{x}}+i v \frac{m}{\hat{l}} \mathcal{C}_{m, n}^{\widehat{u}_{y}}-(1+v) \mathcal{C}_{m, n}^{\varepsilon_{\theta}}\right] \\
\mathcal{C}_{m, n}^{\widehat{\sigma}_{y y}}=\frac{1}{(1+v)(1-2 v)}\left[v \frac{d \mathcal{C}_{m, n}^{\widehat{u}_{x}}}{d \hat{x}}+i(1-v) \frac{m}{\hat{l}} \mathcal{C}_{m, n}^{\widehat{u}_{y}}-(1+v) \mathcal{C}_{m, n}^{\varepsilon_{\theta}}\right] \\
\mathcal{C}_{m, n}^{\widehat{\sigma}_{z z}}=\frac{1}{(1+v)(1-2 v)}\left[v \frac{d \mathcal{C}_{m, n}^{\hat{u}_{x}}}{d \hat{x}}+i v \frac{m}{\hat{l}} \mathcal{C}_{m, n}^{\widehat{u}_{y}}-(1+v) \mathcal{C}_{m, n}^{\varepsilon_{\theta}}\right] \\
\mathcal{C}_{m, n}^{\hat{\sigma}_{x y}}=\frac{1}{2(1+v)}\left(i \frac{m}{\hat{l}} \mathcal{C}_{m, n}^{\widehat{u}_{x}}+\frac{d \mathcal{C}_{m, n}^{\hat{u}_{y}}}{d \hat{x}}\right) .
\end{gathered}
$$

During the undertaken study the solution of (16) - (23) has been received by means of which from (24) - (27) the coefficients of Fourier series of dimensionless stresses have been found. The determined dependences have the form

$$
\mathcal{C}_{m, n}^{\widehat{\vartheta}}=\overline{\mathcal{C}}_{m, n}^{\widehat{\vartheta}} e^{-\left(\xi_{m, n}+i \zeta_{m, n}\right) \hat{x}}, \hat{x} \geq 0, m \in \mathbb{Z}, n \in \mathbb{Z}
$$

$$
\begin{aligned}
& \mathcal{C}_{m, n}^{\widehat{u}_{x}}=\left(\overline{\mathcal{C}}_{m, n}^{\widehat{u}_{x}}+\overline{\mathcal{C}}_{m, n}^{\widehat{u}_{x}} \hat{x}\right) e^{-|m| \hat{x} / \hat{\imath}}+\tilde{\mathcal{C}}_{m, n}^{\widehat{u}_{x}} e^{-\left(\xi_{m, n}+i \zeta_{m, n}\right) \hat{x}}, \hat{x} \geq 0, m \in \mathbb{Z}, n \in \mathbb{Z} ; \\
& \mathcal{C}_{m, n}^{\widehat{u}_{y}}=\left(\overline{\mathcal{C}}_{m, n}^{\widehat{u}_{y}}+\overline{\overline{\mathcal{C}}}_{m, n}^{\widehat{u}_{y}} \hat{x}\right) e^{-|m| \hat{x} / \hat{\imath}}+\tilde{\mathcal{C}}_{m, n}^{\widehat{u}_{y}} e^{-\left(\xi_{m, n}+i \zeta_{m, n}\right) \hat{x}}, \hat{x} \geq 0, m \in \mathbb{Z}, n \in \mathbb{Z} ; \\
& \mathcal{C}_{m, n}^{\widehat{\sigma}_{x x}}=\left(\overline{\mathcal{C}}_{m, n}^{\widehat{\sigma}_{x x}}+\overline{\overline{\mathcal{C}}}_{m, n}^{\widehat{\sigma}_{x x} \hat{x}}\right) e^{-|m| \hat{x} / \hat{l}}+\tilde{\mathcal{C}}_{m, n}^{\widehat{\sigma}_{x x}} e^{-\left(\xi_{m, n}+i \zeta_{m, n}\right) \hat{x}}, \hat{x} \geq 0, m \in \mathbb{Z}, n \in \mathbb{Z} ; \\
& \mathcal{C}_{m, n}^{\widehat{\sigma}_{y y}}=\left(\overline{\mathcal{C}}_{m, n}^{\widehat{\sigma}_{y y}}+\overline{\mathcal{C}}_{m, n}^{\widehat{\sigma}_{y y}} \hat{x}\right) e^{-|m| \hat{x} / \hat{\imath}}+\tilde{\mathcal{C}}_{m, n}^{\widehat{\sigma}_{y y}} e^{-\left(\xi_{m, n}+i \zeta_{m, n}\right) \hat{x}}, \hat{x} \geq 0, m \in \mathbb{Z}, n \in \mathbb{Z} ; \\
& \mathcal{C}_{m, n}^{\widehat{\sigma}_{z Z}}=\left(\overline{\mathcal{C}}_{m, n}^{\widehat{\sigma}_{z Z}}+\overline{\overline{\mathcal{C}}}_{m, n}^{\widehat{\sigma}_{z Z}} \hat{x}\right) e^{-|m| \hat{x} / \hat{\imath}}+\tilde{\mathcal{C}}_{m, n}^{\widehat{\sigma}_{z Z}} e^{-\left(\xi_{m, n}+i \zeta_{m, n}\right) \hat{x}}, \hat{x} \geq 0, m \in \mathbb{Z}, n \in \mathbb{Z} ; \\
& \mathcal{C}_{m, n}^{\widehat{\sigma}_{x y}}=\left(\overline{\mathcal{C}}_{m, n}^{\widehat{\sigma}_{x y}}+\overline{\overline{\mathcal{C}}}_{m, n}^{\widehat{\sigma}_{x y}} \hat{x}\right) e^{-|m| \hat{x} / \hat{l}}+\tilde{\mathcal{C}}_{m, n}^{\widehat{\sigma}_{x y}} e^{-\left(\xi_{m, n}+i \zeta_{m, n}\right) \hat{x}}, \hat{x} \geq 0, m \in \mathbb{Z}, n \in \mathbb{Z} ;
\end{aligned}
$$

where

$$
\begin{aligned}
& \overline{\mathcal{C}}_{m, n}^{\widehat{\vartheta}_{n}}=\mathcal{C}_{m, n}^{\widehat{\vartheta}_{f}} /\left(\frac{\xi_{m, n}}{\mathrm{Bi}}+1+i \frac{\zeta_{m, n}}{\mathrm{Bi}}\right), m \in \mathbb{Z}, n \in \mathbb{Z} ; \\
& \overline{\mathcal{C}}_{m, n}^{\widehat{u}_{x}}=\left\{\begin{array}{l}
0, m=0, n \in \mathbb{Z} ; \\
-(1+v) \frac{\hat{l}}{|m|} \overline{\mathcal{C}}_{m, 0}^{\varepsilon_{\theta}},|m| \in \mathbb{N}, n=0 ; \\
-\frac{(1+v)(1-2 v)}{1-v}\left\{\frac{\zeta_{m, n}}{n}+i\left[\frac{2(1-v)}{1-2 v} \frac{|m|}{n \hat{l}}-\frac{\xi_{m, n}}{n}\right]\right\} \overline{\mathcal{C}}_{m, n}^{\varepsilon_{\theta}},|m| \in \mathbb{N},|n| \in \mathbb{N} ;
\end{array}\right. \\
& \overline{\overline{\mathcal{C}}}_{m, n}^{\hat{u}_{x}}=\left\{\begin{array}{l}
0, m n=0 ; \\
-\frac{1+v}{1-v}\left[\frac{\zeta_{m, n}}{n}+i\left(\frac{|m|}{n \hat{l}}-\frac{\xi_{m, n}}{n}\right)\right] \frac{|m|}{\hat{l}} \overline{\mathcal{C}}_{m, n}^{\varepsilon_{\theta}},|m| \in \mathbb{N},|n| \in \mathbb{N} ;
\end{array}\right. \\
& \tilde{\mathcal{C}}_{m, n}^{\widehat{u}_{x}}=\left\{\begin{array}{l}
0, m \in \mathbb{Z}, n=0 ; \\
-\frac{1+v}{1-v} \frac{1-i \operatorname{sign} n}{\sqrt{2|n|}} \overline{\mathcal{C}}_{0, n}^{\varepsilon_{\theta}}, m=0,|n| \in \mathbb{N} \\
i \frac{1+v}{1-v} \frac{\xi_{m, n}+i \zeta_{m, n}}{n} \overline{\mathcal{C}}_{m, n}^{\varepsilon_{\theta}},|m| \in \mathbb{N},|n| \in \mathbb{N}
\end{array}\right.
\end{aligned}
$$




$$
\begin{aligned}
& \overline{\mathcal{C}}_{m, n}^{\widehat{u}_{y}}=\left\{\begin{array}{l}
0, m=0, n \in \mathbb{Z} ; \\
-i(1+v) \frac{\hat{l}}{m} \overline{\mathcal{C}}_{m, 0}^{\varepsilon_{\theta}},|m| \in \mathbb{N}, n=0 ; \\
\frac{2(1+v)}{\operatorname{sign} m}\left[\frac{1-2 v}{2(1-v)} \frac{|m|}{n \hat{l}}-\frac{\xi_{m, n}}{n}-i \frac{\zeta_{m, n}}{n}\right] \overline{\mathcal{C}}_{m, n}^{\varepsilon_{\theta}},|m| \in \mathbb{N},|n| \in \mathbb{N} ;
\end{array}\right. \\
& \overline{\overline{\mathcal{C}}}_{m, n}^{\widehat{u}_{y}}=\left\{\begin{array}{l}
0, m n=0 ; \\
-\frac{1+v}{1-v}\left(\frac{|m|}{n \hat{l}}-\frac{\xi_{m, n}}{n}-i \frac{\zeta_{m, n}}{n}\right) \frac{m}{\hat{l}} \overline{\mathcal{C}}_{m, n}^{\varepsilon_{\theta}},|m| \in \mathbb{N},|n| \in \mathbb{N} ;
\end{array}\right. \\
& \tilde{\mathcal{C}}_{m, n}^{\widehat{u}_{y}}=\left\{\begin{array}{l}
0, m n=0 ; \\
\frac{1+v}{1-v} \frac{m}{n \hat{l}} \overline{\mathcal{C}}_{m, n}^{\varepsilon_{\theta}},|m| \in \mathbb{N},|n| \in \mathbb{N} ;
\end{array}\right. \\
& \overline{\mathcal{C}}_{m, n}^{\widehat{\sigma}_{x x}}=\left\{\begin{array}{l}
0, m n=0 ; \\
i \frac{1}{1-v} \frac{m^{2}}{n \hat{l}^{2}} \overline{\mathcal{C}}_{m, n}^{\varepsilon_{\theta}},|m| \in \mathbb{N},|n| \in \mathbb{N} ;
\end{array}\right. \\
& \overline{\overline{\mathcal{C}}}_{m, n} \widehat{\sigma}_{x x}=\left\{\begin{array}{l}
0, m n=0 ; \\
\frac{1}{1-v}\left[\frac{\zeta_{m, n}}{n}+i\left(\frac{|m|}{n \hat{l}}-\frac{\xi_{m, n}}{n}\right)\right] \frac{m^{2}}{\hat{l}^{2}} \overline{\mathcal{C}}_{m, n}^{\varepsilon_{\theta}},|m| \in \mathbb{N},|n| \in \mathbb{N} ;
\end{array}\right. \\
& \tilde{\mathcal{C}}_{m, n}^{\widehat{\sigma}_{x x}}=\left\{\begin{array}{l}
0, m n=0 ; \\
-i \frac{1}{1-v} \frac{m^{2}}{n \hat{l}^{2}} \overline{\mathcal{C}}_{m, n}^{\varepsilon_{\theta}},|m| \in \mathbb{N},|n| \in \mathbb{N}
\end{array}\right. \\
& \overline{\mathcal{C}}_{m, n}^{\widehat{\sigma}_{y y}}=\left\{\begin{array}{l}
0, m n=0 ; \\
\frac{1}{1-v}\left[2 \frac{\zeta_{m, n}}{n}+i\left(\frac{|m|}{n \hat{l}}-2 \frac{\xi_{m, n}}{n}\right)\right] \frac{|m|}{\hat{l}} \overline{\mathcal{C}}_{m, n}^{\varepsilon_{\theta}},|m| \in \mathbb{N},|n| \in \mathbb{N} ;
\end{array}\right. \\
& \overline{\overline{\mathcal{C}}}_{m, n}^{\widehat{\sigma}_{y y}}=\left\{\begin{array}{l}
0, m n=0 ; \\
-\frac{1}{1-v}\left[\frac{\zeta_{m, n}}{n}+i\left(\frac{|m|}{n \hat{l}}-\frac{\xi_{m, n}}{n}\right)\right] \frac{m^{2}}{\hat{l}^{2}} \overline{\mathcal{C}}_{m, n}^{\varepsilon_{\theta}},|m| \in \mathbb{N},|n| \in \mathbb{N} ;
\end{array}\right. \\
& \tilde{\mathcal{C}}_{m, n}^{\widehat{\sigma}_{y y}}=\left\{\begin{array}{l}
0, m \in \mathbb{Z}, n=0 ; \\
-\frac{\overline{\mathcal{C}}_{0, n}^{\varepsilon_{\theta}}}{1-v}, m=0,|n| \in \mathbb{N} ; \\
-\frac{1}{1-v}\left(1-i \frac{m^{2}}{n \hat{l}^{2}}\right) \overline{\mathcal{C}}_{m, n}^{\varepsilon_{\theta}},|m| \in \mathbb{N},|n| \in \mathbb{N} ;
\end{array}\right. \\
& \overline{\mathcal{C}}_{m, n}^{\widehat{\sigma}_{z Z}}=\left\{\begin{array}{l}
0, m=0, n \in \mathbb{Z} ; \\
-\overline{\mathcal{C}}_{m, 0}^{\varepsilon_{\theta}},|m| \in \mathbb{N}, n=0 ; \\
\frac{2 v}{1-v}\left[\frac{\zeta_{m, n}}{n}+i\left(\frac{|m|}{n \hat{l}}-\frac{\xi_{m, n}}{n}\right)\right] \frac{|m|}{\hat{l}} \overline{\mathcal{C}}_{m, n}^{\varepsilon_{\theta}},|m| \in \mathbb{N},|n| \in \mathbb{N} ;
\end{array}\right. \\
& \overline{\overline{\mathcal{C}}}_{m, n}^{\widehat{\sigma}_{z Z}}=0, m \in \mathbb{Z}, n \in \mathbb{Z} ; \\
& \tilde{\mathcal{C}}_{m, n}^{\widehat{\sigma}_{z z}}=\left\{\begin{array}{l}
0, m \in \mathbb{Z}, n=0 \\
-\frac{\overline{\mathcal{C}}_{0, n}^{\varepsilon_{\theta}}}{1-v}, m=0,|n| \in \mathbb{N} \\
-\frac{\overline{\mathcal{C}}_{m, n}^{\varepsilon_{\theta}}}{1-v},|m| \in \mathbb{N},|n| \in \mathbb{N}
\end{array}\right. \\
& \overline{\mathcal{C}}_{m, n}^{\widehat{\sigma}_{x y}}=\left\{\begin{array}{l}
0, m n=0 ; \\
\frac{1}{1-v}\left(\xi_{m, n}+i \zeta_{m, n}\right) \frac{m}{n \hat{l}} \overline{\mathcal{C}}_{m, n}^{\varepsilon_{\theta}},|m| \in \mathbb{N},|n| \in \mathbb{N} ;
\end{array}\right.
\end{aligned}
$$




$$
\begin{aligned}
& \overline{\overline{\mathcal{C}}}_{m, n}^{\widehat{\sigma}_{x y}}=\left\{\begin{array}{l}
0, m n=0 ; \\
\frac{\operatorname{sign} m}{1-v}\left(\frac{m}{n \hat{l}}-\frac{\xi_{m, n}}{n}-i \frac{\zeta_{m, n}}{n}\right) \frac{m^{2}}{\hat{l}^{2}} \overline{\mathcal{C}}_{m, n}^{\varepsilon_{\theta}},|m| \in \mathbb{N},|n| \in \mathbb{N} ;
\end{array}\right. \\
& \tilde{\mathcal{C}}_{m, n}^{\widehat{\sigma}_{x y}}=\left\{\begin{array}{l}
0, m n=0 ; \\
-\frac{1}{1-v}\left(\xi_{m, n}+i \zeta_{m, n}\right) \frac{m}{n \hat{l}} \overline{\mathcal{C}}_{m, n}^{\varepsilon_{\theta}},|m| \in \mathbb{N},|n| \in \mathbb{N} ;
\end{array}\right. \\
& \overline{\mathcal{C}}_{m, n}^{\varepsilon_{\theta}}=\Delta \varepsilon_{T_{f}^{*}} \overline{\mathcal{C}}_{m, n}^{\widehat{\theta}}, m \in \mathbb{Z}, n \in \mathbb{Z} ; \\
& \xi_{m, n}=\frac{1}{\sqrt{2}}\left[\left(\frac{m^{4}}{\hat{l}^{4}}+n^{2}\right)^{1 / 2}+\frac{m^{2}}{\hat{l}^{2}}\right]^{1 / 2}, m \in \mathbb{Z}, n \in \mathbb{Z} ; \\
& \zeta_{m, n}=\frac{\operatorname{sign} n}{\sqrt{2}}\left[\left(\frac{m^{4}}{\hat{l}^{4}}+n^{2}\right)^{1 / 2}-\frac{m^{2}}{\hat{l}^{2}}\right]^{1 / 2}, m \in \mathbb{Z}, n \in \mathbb{Z} .
\end{aligned}
$$

From the received dependences it follows that influence of spatial nonuniformity of fluid medium temperature on temperature oscillations and thermocyclic stresses in half-space can be neglected at $\hat{l} \gg 1$. Comparison of the received two-dimensional solution with onedimensional [9] shows that at two-dimensional temperature oscillations there are not only $\sigma_{y y}$ and $\sigma_{z z}$ thermocyclic stresses, but also $\sigma_{x x}$ and $\sigma_{x y}$.

The reported study was funded by RFBR according to the research project № 18-31-00090.

\section{References}

1. H.S. Carslaw, J.C. Jaeger, Conduction of Heat in Solids (Oxford University Press, Oxford, 1959)

2. A.M. Shklover, Heat Transfer under Periodical Cyclical Thermal Effect (Gosenergoizdat, Moscow, 1961) [in Russian]

3. K.F. Fokin, Heat Engineering of Containing Walls of Buildings (AVOK-PRESS, Moscow, 2006) [in Russian]

4. L.B. Getsov, Yu.D. Martynov, Yu.S. Osherov, V.A. Plekhanov, É.P. Rivlin, A.D. Trukhnii, Strength Mater, 2, 1000 (1970)

5. R.Z. Kavtaradze, Local Heat Exchange in Piston Engines (BaumanPress, Moscow, 2016) [in Russian]

6. H. Hausen, Wärmeübertragung im Gegenstrom, Gleichstrom und Kreuzstrom (Springer-Verlag, Berlin, 1976)

7. O. Malyshkina, A. Movchikova, R. Steinhausen, H.T. Langhammer, H. Beige, J. Eur. Ceram. Soc, 30, 211 (2010)

8. O.V. Malyshkina, M.Yu. Gavalyan, G.S. Shishkov, I.A. Kaplunov, A.I. Kolesnikov, N.V. Aidinyan, Phys. Solid State, 58, 2367 (2016)

9. H. Parkus, Instationäre Wärmespannungen (Springer-Verlag, Wien, 1959)

10. E.M. Kartashov, V.A. Kudinov, Analytical Theory of Heat Conduction and Applied Thermoelasticity (Librocom, Moscow, 2012) [in Russian]

11. R.S. Minasyan, J. Eng. Phys. Thermophys., 17, 1396 (1969)

12. Yu.A. Kirsanov, Cyclic Thermal Processes and Theory of Heat Conduction in Regenerative Air Heaters (Fizmatlit, Moscow, 2007) [in Russian]

13. Y.B. Zudin, Theory of Periodic Conjugate Heat Transfer (Springer, Berlin, 2011) 
14. L.K. Martinson, O.Yu. Chigireva, Herald of the Bauman Moscow State Tech. Univ., Nat. Sci., 3, 88 (2015) [in Russian]

15. S.K. Roychoudhuri, Partha Sharathi Dutta, Int J Solids Struct., 42, 4192 (2005)

16. Sadek Hossain Mallik, M. Kanoria, Int J Solids Struct., 44, 7633 (2007)

17. A.E. Green, P.M. Naghdi, J. Elast., 31, 189 (1993)

18. A.G. Shashkov, V.A. Bubnov, S.Y. Yanovsky, Wave phenomena of heat conductivity (Editorial URSS, Moscow, 2004) [in Russian]

19. L. Wang, X. Zhou, X. Wei, Heat Conduction (Springer, Berlin, 2008)

20. A. Jacquot, B. Lenoir, A. Dauscher, M. Stölzer, J. Meusel, J. Appl. Phys., 91, 4733 (2002)

21. M. Ranjbar-Far, J. Absi, G. Mariaux, F. Dubois, Mater. Des., 31, 772 (2010)

22. T.L. Bergman, A.S. Lavine, F.P. Incropera, D.P. DeWitt, Fundamentals of Heat and Mass Transfer (John Wiley \& Sons, Hoboken, NJ, 2011)

23. S.P. Timoshenko, J.N. Goodier, Theory of Elasticity, (McGraw-Hill, NY, 1970)

24. G.P. Tolstov, Fourier Series (Dover Publications, New York, 1976) 